\title{
Data Self-Recalibration and Mixture Mass Fingerprint Searching (DASER-MMF) to Enhance Protein Identification within Complex Mixtures
}

\author{
Ryan M. Danell, ${ }^{\text {a }}$ Severine A. Ouvry-Patat, ${ }^{\mathrm{b}}$ Cameron O. Scarlett, ${ }^{\mathrm{c}, *}$ \\ J. Paul Speir, ${ }^{\mathrm{d}}$ and Christoph H. Borchers ${ }^{\mathrm{b}, \mathrm{e}}$ \\ ${ }^{\text {a }}$ Danell Consulting, Greenville, North Carolina, USA \\ b Department of Biochemistry and Biophysics, University of North Carolina, Chapel Hill, North Carolina, \\ USA \\ c UNC-Duke Proteomics Center, University of North Carolina, Chapel Hill, North Carolina, USA \\ ${ }^{\mathrm{d}}$ Bruker Daltonics, Billerica, Massachusetts, USA \\ e University of Victoria - Genome British Columbia Proteomics Centre, Victoria, British Columbia, Canada
}

A novel algorithm based on Data Self-Recalibration and a subsequent Mixture Mass Fingerprint search (DASER-MMF) has been developed to improve the performance of protein identification from online 1D and 2D-LC-MS/MS experiments conducted on high-resolution mass spectrometers. Recalibration of $40 \%$ to $75 \%$ of the MS spectra in a human serum dataset is demonstrated with average errors of $0.3 \pm 0.3 \mathrm{ppm}$, regardless of the original calibration quality. With simple protein mixtures, the MMF search identifies new proteins not found in the MS/MS based search and increases the sequence coverage for identified proteins by six times. The high mass accuracy allows proteins to be identified with as little as three peptide mass hits. When applied to very complex samples, the MMF search shows less dramatic performance improvements. However, refinements such as additional discriminating factors utilized within the search space provide significant gains in protein identification ability and indicate that further enhancements are possible in this realm. (J Am Soc Mass Spectrom 2008, 19, 1914-1925) (C) 2008 American Society for Mass Spectrometry

$\mathrm{T}$ The multidimensional protein identification technology (MudPIT), consisting of a 2D liquid separation of the peptides followed by mass spectrometric sequencing and database search is likely the most popular technique to identify proteins within a complex mixture [1]. Unfortunately, protein identifications still depend on matching experimental and predicted tandem mass spectra of several peptides per protein, and under-sampling of peptides is an issue [2]. Factors such as low MS/MS duty cycle, peptide abundances, ionization efficiency, chromatographic resolution, sample complexity, and ion suppression affect this random sampling phenomenon [3]. Repeated analyses of the same sample can partially overcome this undersampling problem but it is a time consuming process [3].

The accurate mass tag (AMT) strategy offers an alternative approach to complex mixture analysis. The AMT approach involves extensive proteome coverage of a particular sample by bottom up analysis followed by exact mass measurements to create a database of

Address reprint requests to Dr. C. Borchers, UVic Genome BC Proteomics Centre, Victoria, BC, Canada. E-mail: Christoph@proteincentre.com

* Current address: School of Pharmacy, University of Wisconsin-Madison, Madison, WI 53705, USA. these mass tags. The original AMT implementation used only MS/MS data to create the mass tag database $[4,5]$, but more recently chromatographic retention times have been added to increase discrimination capabilities [6]. While this strategy allows for subsequent high throughput MS analysis on an accurate mass instrument, the creation of the AMT database for a particular proteome is time consuming [5] and requires extremely reproducible chromatographic separations. Interesting proteins are often of lower abundance and may therefore not be selected due to random sampling, even though most peptide $\mathrm{m} / \mathrm{z}$ values will be measured in the MS spectra. Indeed, using a strategy based solely on database searching with accurate masses, Goodlett et al. were able to identify low abundance proteins from yeast cell lysates that were not identified by traditional tandem mass spectrometry [7].

To perform database searches without tandem mass spectra in a 2D-LC dataset, high mass accuracy is a requirement. Fourier transform ion cyclotron resonance (FTICR) instrumentation can achieve high mass accuracy (low to sub-ppm) and ultra-high-resolution $(m / \Delta m$ about 100,000) under the right conditions [8]. The main limitation to high accuracy is space charge effects in the ICR cell $[9,10]$. To overcome this space charge effect, the 
spectra have to be carefully calibrated. External calibration is the most common and straight forward method; however, it cannot provide high mass accuracy with an online HPLC separation as the space charge in the cell will vary with the concentration of the eluted peptides. Automatic gain control can be used to control the number of ions in the ICR cell resulting in improved mass accuracy but it also results in a decrease in dynamic range [11-13]. Internal calibration is largely considered the best calibration method as the calibrants and the analytes will be exposed to the same space charge conditions in the ICR cell [14, 15]. However, internal calibration has its own set of complications, including ion suppression of the calibrants or analytes, increased space charge in the ICR cell from the calibrants, and possibly complex hardware [14, 16-18]. Although several methods to recalibrate spectra without internal or external calibrants have been developed, none are suitable for 2D-LCMS datasets [19-22]. Tolmachev et al. developed a multidimensional calibration technique that separates the peaks in the dataset by total ion abundance, individual peak intensity, peak $\mathrm{m} / \mathrm{z}$ value, and LC retention time, and uses a statistical analysis of peak matches to accurate masses (i.e., from a list of PMT tags or an in-silico generated list) to recalibrate the entire dataset [23]. While very good mass accuracy and precision improvements have been observed, this technique relies on the availability of the detailed list of putative compounds.

We have developed a 2D-LC tandem mass spectrometrybased recalibration strategy similar to the disclosed by Palmblad et al. [24]. These methods use identifications from MS/MS data to self-recalibrate (DASER) as many MS spectra as possible. As shown here, the resulting peaks lists typically have average mass accuracies below $1 \mathrm{ppm}$. The accurate mass peaks are then searched with a custom mixture mass fingerprint (MMF) search algorithm to yield additional protein identifications. Therefore, we have named this combined method DASER-MMF. The ultimate goal of these further developments is to preserve the maximum amount of instrument dynamic range and provide efficient calibration of spectra to improve the protein identification performance of high-resolution mass spectrometers.

Here we extend the Palmblad et al. recalibration technique to very complex mixtures (human serum) and observe recalibration performance similar to that previously reported (with some improvements likely due to field strength). While our recalibration technique is very similar to that previously described (we do not observe or use the polydimethylcyclosiloxane contaminant ions observed in their study) [24], the way that we use the calibrated masses is quite different. The MMF search presented here has more resemblance to an early report from the same laboratory [25]. Similar to the likelihood ratios used by Ramström et al., the MMF search presented here relies on the fact that peptides will occur in the dataset as a collection all stemming from the same protein. If a large enough collection of peptide masses is found in the dataset, the masses will mutually support each other and positively identify the protein. Additionally, by identifying multiple species associated with a single protein, the MMF search has the potential to be interfaced with quantitation techniques for comparing protein expression. In this work, we use a novel scoring function and apply this search scheme to a very large database that is not speciesspecific. This is the first report of identifying a mixture of proteins using accurate mass alone and a search against a large (over one million sequences) sequence database.

\section{Experimental}

\section{Materials}

Mass spectrometry grade Chromasolv solvents and HPLC grade solvents were obtained from Sigma (St. Louis, MO) and Fisher Scientific (Fair Lawn, NJ), respectively. Angiotensin for external calibration along with acetic and hexadeuteroacetic anhydride were also acquired from Sigma. A six-protein tryptic digest mixture (BSA, transferrin, cytochrome $c, \beta$-galactosidase, lysozyme, and alcohol dehydrogenase) was purchased from Dionex (Sunnyvale, CA). Tris(2-carboxyethyl) phosphine hydrochloride (TCEP), methyl methanethiosulfonate (MMTS), and trifluoroacetic acid (TFA) were acquired from Pierce (Rockford, IL).

\section{Protein Digest and Labeling}

The Dionex six-protein tryptic digest mixture was resuspended to a concentration of $10 \mathrm{fmol} / \mu \mathrm{L}$ with loading buffer (water:acetonitrile:TFA; 98:2:0.03) and used directly $(5 \mu \mathrm{L}=50 \mathrm{fmol}$ and $15 \mu \mathrm{L}=150 \mathrm{fmol}$ ). Serum samples from a cystic fibrosis (CF) patient before and after antibiotic treatment, which were chemically labeled and then mixed, were used as a more complex system. Proteins within the serum were reduced with 5 $\mathrm{mM}$ TCEP and alkylated with $10 \mathrm{mM}$ MMTS and digested with trypsin overnight. The peptides were labeled with either acetic anhydride (pre-antibiotic treatment) or hexadeuteroacetic anhydride (post-treatment) anhydride for $10 \mathrm{~min}$ followed by two or four more additions of anhydride with $10 \mathrm{~min}$ incubations using at least a 50-fold molar excess. The peptides were lyophilized to dryness and then resuspended in 5\% acetonitrile: $0.1 \%$ formic acid to a final concentration of $1 \mu \mathrm{g} / \mu \mathrm{L}$. Heavy and light labeled peptides were then mixed $1: 1$. Five $\mu \mathrm{L}$ of the prepared samples (containing a total of $5 \mu \mathrm{g}$ of serum proteins) were loaded on the 2D-LC.

\section{Liquid Chromatography and Mass Spectrometry}

The 2D-LC system consisted of a strong cation exchange capillary column (Poros S10, $300 \mu \mathrm{m} \times 15 \mathrm{~cm}$; Dionex, Sunnyvale, CA) followed by a reverse phase nano- 
column (C18 PepMap100, $75 \mu \mathrm{m} \times 15 \mathrm{~cm}, 3 \mu \mathrm{m}, 100 \AA$ 凡; Dionex) mounted on an Ultimate 3000 LC instrument (Dionex). For the 2D-LC experiments, salt plugs were used to elute the peptides bound to the cation exchange column. Eleven salt fractions were programmed: 0 (flow through), 1, 2, 5, 10, 25, 50, 100, 200, 500, and 1000 $\mathrm{mM} \mathrm{NaCl}$. For 1D-LC experiments, the strong cation exchange capillary column was bypassed. Two percent acetonitrile with $0.03 \%$ TFA was used to load the sample from the injection loop to the capillary column (2D configuration) or to the trap column (1D configuration) at a flow rate of $20 \mu \mathrm{L} / \mathrm{min}$. This solvent was also used to load the salt plugs onto the capillary column and to desalt the peptides loaded on the trap column (samples from 1D injections or peptides eluted from the cation exchange column during 2D injections). The solvents used to elute the peptides bound to the reverse-phase nano-column consisted of $0.1 \%$ formic acid (Solvent A) and 90\% acetonitrile, $0.1 \%$ formic acid (Solvent B). The reverse-phase gradient was $4 \%$ to $55 \%$ Solvent B run over $60 \mathrm{~min}$ at a flow rate of $300 \mathrm{~nL} / \mathrm{min}$. After each elution the column was washed (gradient to $100 \%$ Solvent B) and equilibrated (4\% Solvent B). The output from the LC system was connected directly to the electrospray source of the mass spectrometer.

Mass spectra (MS or MS/MS) were acquired on an Apex-Qe (Qh-FTICR) mass spectrometer (Bruker Daltonics, Billerica, MA) equipped with a 12-tesla actively shielded magnet and an Apollo II (Bruker Daltonics) electrospray ionization source. The instrument was configured to operate in auto-MS/MS mode, selecting one precursor from each MS scan to automatically dissociate in the external collision cell. The overall acquisition rate was between 3 and $4 \mathrm{~s}$ per scan (MS or MS/MS) and $512 \mathrm{k}$ data points were collected in each acquisition. When raw data were collected, no signal/ spectral averaging was performed. The instrument was calibrated externally before each LC-MS/MS analysis with an angiotensin $(0.001 \mathrm{mg} / \mathrm{mL})$ CID spectrum.

\section{MS/MS Database Search}

The recalibration procedure centers around the results from an MS/MS based database search and identification. For all of the work presented here, the Mascot (Matrix Science, London, UK) search engine was used; however, the technique and concept are compatible with any search engine capable of identifying analytes from MS/MS spectra. The mass error is an important parameter in the search. This value has to be large enough to account for the possibly poor initial calibration of the instrument and/or the calibration issues within a single spectrum due to spray conditions, space charge, etc. Typically, searches are conducted with a rather high mass error (for a given instrument) so that as many MS/MS spectra as possible are identified, which will lead to enhanced recalibration results. The exact search parameters for each MS/MS ion search used in this work will be given in the discussion of those search results.

\section{Databases}

Two protein sequence databases were used in this work for both the Mascot MS/MS ion search and the MMF search. For searches on the simple protein mixture data, the complete MSDB database was used. The database contains 2,344,227 protein sequences and all of these were considered, i.e., no species filter was implemented. For searches on serum samples from CF patients the NCBI nonredundant protein database was used. In this case, the sequences were filtered such that only human sequences were considered, yielding a total of 145,700 protein sequences.

\section{Results and Discussion}

\section{DASER Algorithm}

The DASER-MMF method developed in this work involves several steps of post-processing after the LC-MS/MS data has been collected. The first step is to perform an initial Mascot MS/MS ion search, the results of which are used as input to the DASER algorithm. A detailed flow chart of the DASER algorithm we developed and implemented in custom software is shown in Figure 1. While the general operation of the recalibration procedure is similar to that reported by Palmblad et al. [24], there are some differences in our specific implementation, hence a description of our entire process is given here. Recalibration is generally performed on averaged spectra (MS only) from the LC dataset. Average times depend on the chromatographic parameters and resulting peak widths, but typically an averaging width of $3 \mathrm{~min}$ is used. The algorithm implements a boxcar average with $50 \%$ overlap so that every MS spectrum appears in two average spectra. Chromatographic peak widths on our system are usually in the 1 to 2 min range, so a boxcar average width of $3 \mathrm{~min}$ means that most LC peaks will be completely contained in one of the average spectra. Averaging the spectra like this means that a greater portion of the data (more individual spectra) will be recalibrated, and also im-

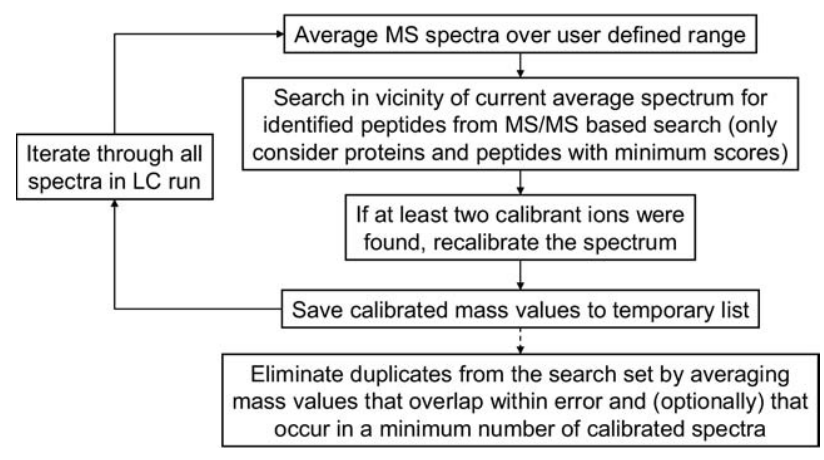

Figure 1. Detailed flow chart of DASER method. 
proves FTMS peak shape making isotopic distribution identification and mass determination more accurate. The raw spectra are initially processed within Data Analysis (DA; Bruker Daltonics, Billerica, MA). The boxcar averaging algorithm is implemented in a Visual Basic for Applications (VBA) script within DA and the SNAP2 algorithm is used to identify monoisotopic peaks. SNAP2 uses an advanced isotopic distribution fitting routine to find monoisotopic masses even in cases when several distributions overlap. Identified peptides from the MS/MS search results are used as calibrant ions. Only peptides from proteins with scores above the confidence limit for that given search are used in the recalibration scheme. A second filter on the actual peptide score (this is typically set at 10) is used to further discriminate against peptides that were not confidently identified based on their MS/MS spectrum. In this way, the ions used to recalibrate a given experiment have been validated based on their MS/MS spectrum as well as the fact that they exist as a collection, not a single entity.

Once the list of calibrant peptide masses has been filtered, the monoisotopic peak list from each spectrum is searched for these identified masses. Because the observation of each ion will be characterized by a chromatographic profile, a given calibrant ion is only considered valid in the local vicinity (in time) of where its MS/MS identification occurred. The mass value in a given spectrum must also be within a defined error (typically the same error as was used for the MS/MS search to find the calibrant ions) of the calibrant mass for it to be considered the same species and to be used in the calibration. Assuming at least two calibrant ions are found in a given spectrum, that spectrum is recalibrated using a linear fit based on the following frequency to $m / z$ relationship (this is called the "CAL2" function by Bruker, but is previously attributed to Francl et al. [26]):

$$
m / z=\frac{A}{f+B}
$$

The observed ion frequencies are first calculated using the original calibration constants $(A$ and $B)$ and solving the above equation for $f$, the measured cyclotron frequency. These frequencies, along with the calibrated $\mathrm{m} / \mathrm{z}$ values, are then used to find new calibration constants using a least-squares minimization fitting algorithm. Ideally, one would like to have more than two points in the calibration so that a more representative fit to the actual data could be generated. For many spectra this is the case; however, in an effort to recalibrate as many spectra as possible, spectra where only two calibrants could be found were still accepted. For FTMS data it is also possible to perform a single point space charge calibration [27]. Due to the nature of the identifications being made in this work and the need for ultimate accuracy, we chose not to investigate this single point calibration mode.
Before any recalibrations are accepted, a final filter on the average error of the calibrant ions is applied. This filter is used to address issues where ion signals are incorrectly identified as calibrant ions in a given spectrum and lead to erroneous calibrations and, therefore, erroneous calibrated mass values. If the average error for the calibrants after the recalibration is greater than a user defined limit (2 ppm was used for all data presented here), that calibration is considered flawed and peaks from that spectrum are not used for further analysis. It should be noted that every effort is made to only include high quality, accurate calibrations in this analysis so that the results reflect the highest mass accuracy obtainable from the instrument.

After a given spectrum recalibration has been deemed acceptable, the entries in its accurate mass peak list are added to a temporary mass list. Due to the elution profiles of analytes and the spectral averaging used, masses may occur in the list several times as they will be observed in multiple recalibrated spectra. A final unique mass value algorithm is used to sort through this list and find mass values that are within a defined error of one another. This error is now the average calibrated error of all the spectra, which is much smaller than the errors used in the steps above. For all the data presented here, a recalibration error of 2 ppm was used to ensure that all similar masses could be grouped. The unique mass value algorithm centers on a histogram analysis of the data, allowing similar masses to be easily identified and their mass values averaged. In this way, a single list of unique masses from the entire experiment is generated for input to the MMF search. A key point here is that this list contains mass values from averaged calibrated spectra that were then averaged with additional calibrated values providing very accurate masses based on multiple measurements. The result, as supported by the actual errors found during the MMF search (see results below), is a mass list with sub-ppm accuracy.

\section{MMF Search}

The mixture mass fingerprint search consists of a search through a protein database looking for peptide masses that match masses contained in the unique and accurate mass list produced from the DASER method. As its name implies, the MMF search is based on the well established peptide mass fingerprint (PMF) search [28]. The key difference is that the MMF search is designed, based both on the quality of the input data as well as the operation and scoring scheme, to handle a mass list from a mixture of different proteins. The use of recalibrated, high accuracy data greatly reduces the ambiguity in identifying a mass as being from a particular protein. Additionally, a high quality separation means that more peptides from a given protein will be observable and not masked by other more intense ions. Therefore, a real protein hit is expected to match several peptides leading to significant sequence coverage (com- 
monly $15 \%$ or greater). The accuracy combined with the high sequence coverage is what allows a protein to be unambiguously identified within a mixture. Database size, complexity, and occurrence of proteins with significant homology will present the biggest hurdles to successful application of this technique. As shown in the examples below, real databases do not present unmanageable complexity, and confident protein hits can be identified within a mixture. Finally, it is implicitly difficult for this algorithm to identify small proteins that produce only a few digest fragments. This cutoff, while not yet analyzed in detail, appears to occur at $\sim 100$ residues. Proteins that are smaller than this may not produce enough detectable peptides to confidently identify them above the noise threshold of the scoring technique. The protein sequence as well as the sample and database complexity will all have some effect on where this performance cutoff appears. MS/MS data is likely required to identify small proteins within a mixture.

The MMF search starts by performing an in-silico digest (and modification as necessary) of each protein in the database. If any of the calculated digest masses match a mass in the accurate list, again within a specified error (the same calibrated error of 2 ppm was used for all searches here), then this match is recorded for subsequent scoring at the end of the search. After all proteins in the database have been searched the individual protein hits are scored. The protein score is calculated using the following empirically determined function:

$$
\text { score }=\log \left(N P M M^{4} \times A S C^{2} \times A M I\right)
$$

The number of peptide mass matches (NPMM) is simply the number of peptides matching to that protein. The adjusted sequence coverage (ASC) is a measure of the sequence coverage with every peptide hit only counting once. This is important for proteins that have repeat subunits as a single peptide match can lead to almost $100 \%$ coverage, however, that is clearly not as confident a hit as one made up of five unique peptide matches leading to $20 \%$ coverage. The average match intensity $(A M I)$ is an average of the peak intensities for the peptide mass matches with the highest and lowest intensities discarded. Discarding the high and low intensities eliminates any biasing in this value from possibly anomalous matches; it also means that the minimum value for NPMM is three (discarding two intensities leaves one value, which becomes the $A M I$ ). As can be seen, the scoring function weights the number of peptide matches as well as the sequence coverage fairly heavily as these two values are implicitly what MMF identifications are based on. The inclusion of the average match intensity makes the score an arbitrary value as each dataset will exhibit different peak intensities based on the sample and ion source conditions. The peak intensity was included in the score to help discriminate between matches to very low intensity peaks that may be due to noise or contaminants. Acceptable performance was observed using a scoring function that did not include the match intensity; however, small species that produce few fragment ions within a mixture could not be identified and therefore this component was added to the score. For attempting to identify low concentration components, a scoring function that does not include intensity may be desirable and will be investigated in the future, as will the inclusion of other factors, such as the MOWSE influence [29]. The score is log scaled for easier comparison and visualization, but it should be remembered that even a tenth of a point is a large difference in the raw score.

As the score is an arbitrary value, methods are needed to confidently determine what value is significant and, therefore, which proteins are positively identified as being present in the sample. The first method employed involves converting the scores into expectation values using a previously described technique [30]. This calculation is based on a survival function constructed from the score distribution of protein hits to the database. While the expectation values can give a good indication of the quality of the top hits, we have found that they perform poorly in identifying proteins close to the significance cutoff. The second method involves a second search on a decoy protein database. The decoy database is produced by randomizing each individual sequence in the original database, therefore producing a new set of digest masses but not altering the overall amino acid composition of a given protein. The score distribution of hits to the decoy database is fit to the probability density function of a suitable distribution. The false positive rate associated with a given score can then be calculated using the cumulative distribution function for the distribution as all hits to the decoy database are assumed false. This analysis is similar to that implemented within the ProteinProphet package and, similar to their conclusions, we also find that a $\gamma$ distribution best models the decoy database score distribution [31]. Based on the simple protein mixture results shown below, false positive rates below $0.3 \%$ to $0.5 \%$ are generally required for accurate protein identification with the MMF search.

\section{Simple Protein Mixture}

Two different column loadings (50 and $150 \mathrm{fmol}$ of each protein) of the six-component protein mixture were each analyzed three separate times by LC-MS/MS. The data from each experiment was exported to an MGF file and submitted to Mascot for an MS/MS ion search (MSDB database, no species filter, trypsin cleavage rules, two missed cleavages, carboxymethyl (C) variable modification, 10 ppm mass accuracy). These results were then used to recalibrate the data using the DASER algorithm. A complete summary of the recalibration results is shown in Table 1 . These datasets averaged slightly more than $20 \%$ recalibration coverage with the higher column loadings producing over $30 \%$ coverage. 
Table 1. Recalibration results from simple protein mixture datasets

\begin{tabular}{|c|c|c|c|c|c|c|c|c|c|c|}
\hline \multirow[b]{2}{*}{ Dataset } & \multirow[b]{2}{*}{$\begin{array}{l}\text { No. MS/MS } \\
\text { spectra }\end{array}$} & \multirow[b]{2}{*}{$\begin{array}{c}\% \text { MS/MS } \\
\text { spectra IDed }\end{array}$} & \multirow[b]{2}{*}{$\begin{array}{c}\% \text { Data } \\
\text { recalibrated }\end{array}$} & \multicolumn{6}{|c|}{ Errors } & \multirow[b]{2}{*}{$\begin{array}{l}\text { Unique } \\
\text { masses }\end{array}$} \\
\hline & & & & Orig RMS & $\begin{array}{l}\text { Recal } \\
\text { RMS }\end{array}$ & $\begin{array}{c}\text { Orig } \\
\text { mean }\end{array}$ & $\begin{array}{l}\text { Recal } \\
\text { mean }\end{array}$ & Orig STD & $\begin{array}{l}\text { Recal } \\
\text { STD }\end{array}$ & \\
\hline \multicolumn{11}{|l|}{$50 \mathrm{fmol}$} \\
\hline 1 & 76 & $28 \%$ & $24 \%$ & 0.77 & 0.32 & 0.39 & 0.00 & 0.58 & 0.35 & 1082 \\
\hline 2 & 24 & $25 \%$ & $13 \%$ & 1.08 & 0.04 & -1.04 & 0.00 & 0.30 & 0.05 & 340 \\
\hline 3 & 22 & $18 \%$ & $6 \%$ & 1.26 & 0.00 & -1.25 & 0.00 & 0.13 & 0.00 & 169 \\
\hline \multicolumn{11}{|l|}{$150 \mathrm{fmol}$} \\
\hline 4 & 104 & $15 \%$ & $19 \%$ & 0.83 & 0.39 & 0.51 & 0.00 & 0.57 & 0.44 & 1133 \\
\hline 5 & 106 & $25 \%$ & $30 \%$ & 1.61 & 0.25 & 1.55 & 0.00 & 0.37 & 0.27 & 1338 \\
\hline 6 & 96 & $27 \%$ & $32 \%$ & 1.06 & 0.23 & 0.98 & 0.00 & 0.40 & 0.25 & 1437 \\
\hline Averages & & $23 \%$ & $21 \%$ & 1.10 & 0.21 & 0.19 & 0.00 & 0.39 & 0.23 & \\
\hline
\end{tabular}

Variability in the instrument performance (likely LC efficiency and ion source optimization) affected the specific results. These samples exhibited somewhat lower than normal percentage recalibration due to the mixture simplicity and general lack of peptides present to identify and use for recalibration.

The root mean squared (RMS), mean, and standard deviation (STD) of the mass error before and after recalibration is also shown in Table 1 . The RMS error gives a good indication of the true "average error" of a dataset as one spectrum with a +10 ppm error and another with a -10 ppm error will yield a mean error of 0 ppm, while the RMS error for these spectra is 10 ppm. The errors are calculated from the calibrant ions identified and used in each spectrum within the dataset. Therefore, this is not a completely unbiased measurement of the actual error of the data. Generally, these computed values will underestimate the real error; however, they still serve to indicate the improvement observed with the recalibration scheme. An unbiased analysis of the errors in the peptide masses identified by the MMF search is presented below. Although these datasets generally exhibited fairly low errors before processing (0.8-1.6 ppm), the recalibration errors all show significant improvement. Specifically, the RMS and standard deviation of the errors are all below 0.5 ppm, with several below $0.25 \mathrm{ppm}$. These errors indicate that database searches can be confidently performed on the 1 to 2 ppm level.

The accurate mass lists from the recalibrated datasets were submitted to the mixture mass fingerprint search (MSDB database, no species filter, Trypsin cleavage rules, two missed cleavages, carboxymethyl (C) variable modification, 2 ppm mass error). The complete results of the Mascot and MMF searches on dataset no. 4 (a representative dataset from the six presented above) are shown in Table 2. While there was a reasonable number of MS/MS spectra included in the Mascot search, two of the proteins present in the sample were not identified in the search (alcohol dehydrogenase and cytochrome c). The incomplete identification via MS/MS data is likely due to the MS/MS acquisition rate and the column loading (150 fmol) used with this sample. The MMF search, however, is able to positively identify all six proteins contained in the mixture. The MMF search makes these identifications with an aver-

Table 2. Mascot and MMF search results from protein mixture dataset no. 4, $150 \mathrm{fmol}$ of each protein loaded on the column

\begin{tabular}{|c|c|c|c|c|c|c|c|c|c|c|c|c|}
\hline \multirow[b]{2}{*}{ Accession } & \multirow[b]{2}{*}{$\begin{array}{c}\text { Protein } \\
\text { description }\end{array}$} & \multicolumn{4}{|c|}{ Mascot } & \multicolumn{5}{|c|}{ MMF } & \multirow[b]{2}{*}{$\begin{array}{l}\text { Seq cov } \\
\text { increase }\end{array}$} & \multirow{2}{*}{$\begin{array}{l}\text { Seq cov } \\
\text { fold } \\
\text { increase }\end{array}$} \\
\hline & & Rank & Score $^{a}$ & $\begin{array}{l}\text { Total } \\
\text { hits }\end{array}$ & $\begin{array}{l}\text { Seq } \\
\text { cov }\end{array}$ & Rank & Score $^{b}$ & $\begin{array}{l}\text { Exp } \\
\text { value }\end{array}$ & $\begin{array}{c}\text { Total } \\
\text { hits }\end{array}$ & Seq cov & & \\
\hline AAN17824 & $\begin{array}{l}\text { Bos taurus } \\
\text { serum albumin }\end{array}$ & 1 & 278 & 5 & 8 & 1 & 14.6 & 0.00032 & 36 & 46 & 38 & 4.8 \\
\hline AAA96735 & $\begin{array}{l}\text { Bos taurus } \\
\text { transferrin }\end{array}$ & 2 & 221 & 5 & 8 & 2 & 14.3 & 0.00067 & 32 & 37 & 29 & 3.6 \\
\hline $\mathrm{LZCH}$ & $\begin{array}{l}\text { Lysozyme } c \\
\text { precursor }\end{array}$ & 3 & 113 & 2 & 19 & 4 & 12.1 & 0.15 & 8 & 48 & 29 & 1.5 \\
\hline GBEC & $\beta$-Galactosidase & 4 & 49 & 1 & 1 & 5 & 11.9 & 0.22 & 17 & 15 & 14 & 14.0 \\
\hline DEBYA & $\begin{array}{l}\text { Alcohol } \\
\text { dehydrogenase }\end{array}$ & - & 0 & 0 & 0 & 3 & 12.2 & 0.14 & 14 & 33 & 33 & $\operatorname{lnf}$ \\
\hline ССВО & $\begin{array}{l}\text { Cytochrome } c \\
\text { bovine }\end{array}$ & - & 0 & 0 & 0 & 6 & 11.0 & 2.2 & 5 & 36 & 36 & $\operatorname{lnf}$ \\
\hline \multicolumn{4}{|c|}{$\begin{array}{l}\text { Total Hits/average seq cov (including } 0 \text { s) } \\
\text { Fold increase }\end{array}$} & 13 & 6 & & & & $\begin{array}{l}112 \\
8.6\end{array}$ & $\begin{array}{l}36 \\
6.0\end{array}$ & & \\
\hline
\end{tabular}

a Significance cutoff for the Mascot search was a score of $38(P<0.05)$.

bignificance cutoff for the MMF search was a score of $10.8(0.3 \%$ false positive rate). 
age of almost nine times as many peptides per protein, yielding six times the sequence coverage per protein. The analysis of the decoy search on a randomized protein database created from the entries in the MSDB yielded a false positive rate of $0.15 \%$ at a protein score of 11.0. While this false positive rate could be manually chosen to correctly identify all the species present in this known sample, even at a rate of $0.3 \%$ (found to be an acceptable level for the MMF search based analysis of several known samples) all the correct proteins are identified and no incorrect IDs are generated. The expectation values for the protein IDs shown in Table 2 indicate that the top five proteins are quite confident identifications. The expectation value for cytochrome $c$, however, indicates that is it no better than a stochastic match even though its score is above the $0.15 \%$ false positive cutoff. Cytochrome $c$ is an example of a small protein that is difficult to identify within a mixture. While the MMF search is able to achieve $36 \%$ sequence coverage on this protein, only five peptides provide this coverage. The discrepancy between the number of matching peptides and the sequence coverage illustrates the source of the uncertainty problem with small proteins. This case also highlights the apparent issues with using this expectation value calculation method [30] when trying to identify proteins near the significance cutoff. Finally, it should be pointed out that the above performance was achieved when searching the entire MSDB database of over two million protein sequences, a significant enhancement over the previous report that restricted the search to considerably fewer sequences [24]. At a false positive rate of $0.15 \%$, our method was able to identify all of the components in this mixture, and no others, despite the fact that only peptide mass was used to identify proteins.

The results from the MMF search allow the actual errors present in the dataset to be analyzed in an unbiased way. Figure 2 shows a histogram of the errors for each peptide match identified by the MMF search. The errors are centered very close to 0 ppm and exhibit a very narrow distribution (standard deviation $=0.56$ $\mathrm{ppm}$ ). The RMS error for these matches, $0.57 \mathrm{ppm}$, gives a good indication of the actual calibrated mass accuracy of the entire dataset.

The MMF search also allows the performance of the DASER technique to be analyzed in more detail. Figure 3 shows the result of this analysis on a peak within the protein mixture dataset no. 4. This peak was one of many peaks identified by the MMF search but not via MS/MS analysis. The MMF search identified this peak as originating from a peptide, which is from a protein that was positively identified by the Mascot MS/MS ion search. Therefore, the actual presence of this peptide in the sample is very likely. Figure 3 a shows an averaged mass spectrum over a 3 min observation time (44 total individual spectra, a typical average time for processing, as noted above). The six calibrant ions identified via MS/MS analysis are marked in the spectrum. The peak marked with an arrow was identified by the MMF

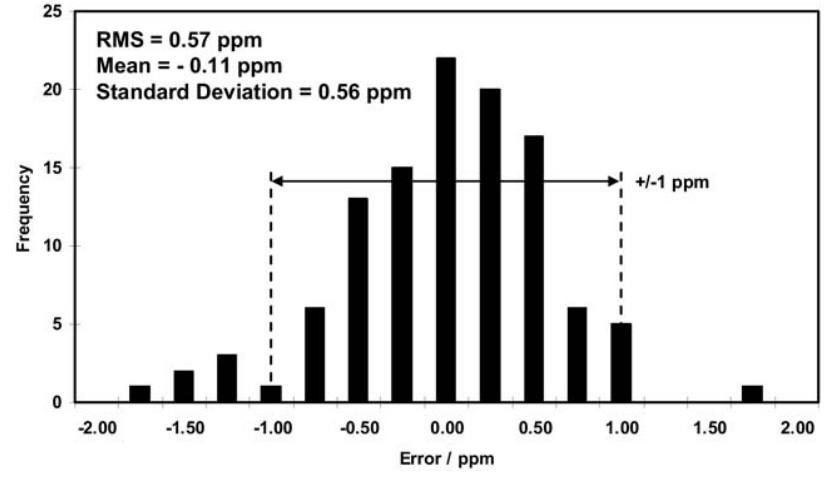

Figure 2. Error histogram for MMF peptide matches from protein mixture dataset no. 4. Mass errors for each individual peptide match identified by the MMF search provided the 112 data points. The MMF search was completed considering mass errors up to 2 ppm.

search as the $2+$ ion of an 11 residue peptide from BSA (sequence: HLVDEPQNLIK). Figure $3 b$ shows the detailed mass measurement accuracy analysis on this $2+$ ion. A perfect measurement of the mass would be at 0 ppm (line marked " $\mathrm{A}$ "). Open bars are a histogram of the individual mass values obtained from all raw mass spectra (a total of 101) where this isotopic distribution was observed. The line marked " $\mathrm{B}$ " is the average of these values. Shaded bars are a histogram of the mass values obtained from the averaged and recalibrated mass spectra (a total of 6) produced from the DASER analysis. The line marked " $C$ " is the average of these values. The average mass value obtained from the raw spectra is 1.2 ppm from the theoretical peptide mass, whereas the average mass obtained from the DASER analysis only has an error of $0.1 \mathrm{ppm}$. Similar performance was observed with multiple other peaks investigated regardless of whether or not their parent protein was identified by the original Mascot MS/MS ion search (data not shown). This analysis shows that the resulting mass measurements made after the DASER analysis are not only centered on the theoretical peptide mass but they also have a smaller overall spread in measured mass; that is, both the accuracy and the precision of the measurement are improved.

The ultimate performance obtainable with the MMF search can be seen by looking at the results from an analysis with very few Mascot identifications. The complete results of the Mascot and MMF searches on dataset no. 2 are shown in Table 3. This dataset was generated from a lower column loading (50 fmol on column) than the previous example, and apparently the instrument source and MS/MS parameters were not optimized as well, yielding very few MS/MS identifications via Mascot. Only two proteins were confidently identified via Mascot, giving only four calibrant ions. However, enough of the dataset was recalibrated (13\%, see Table 1) to allow for the confident identification of five of the six proteins present in the mixture at a $0.3 \%$ false positive rate. Overall improvements in the number 

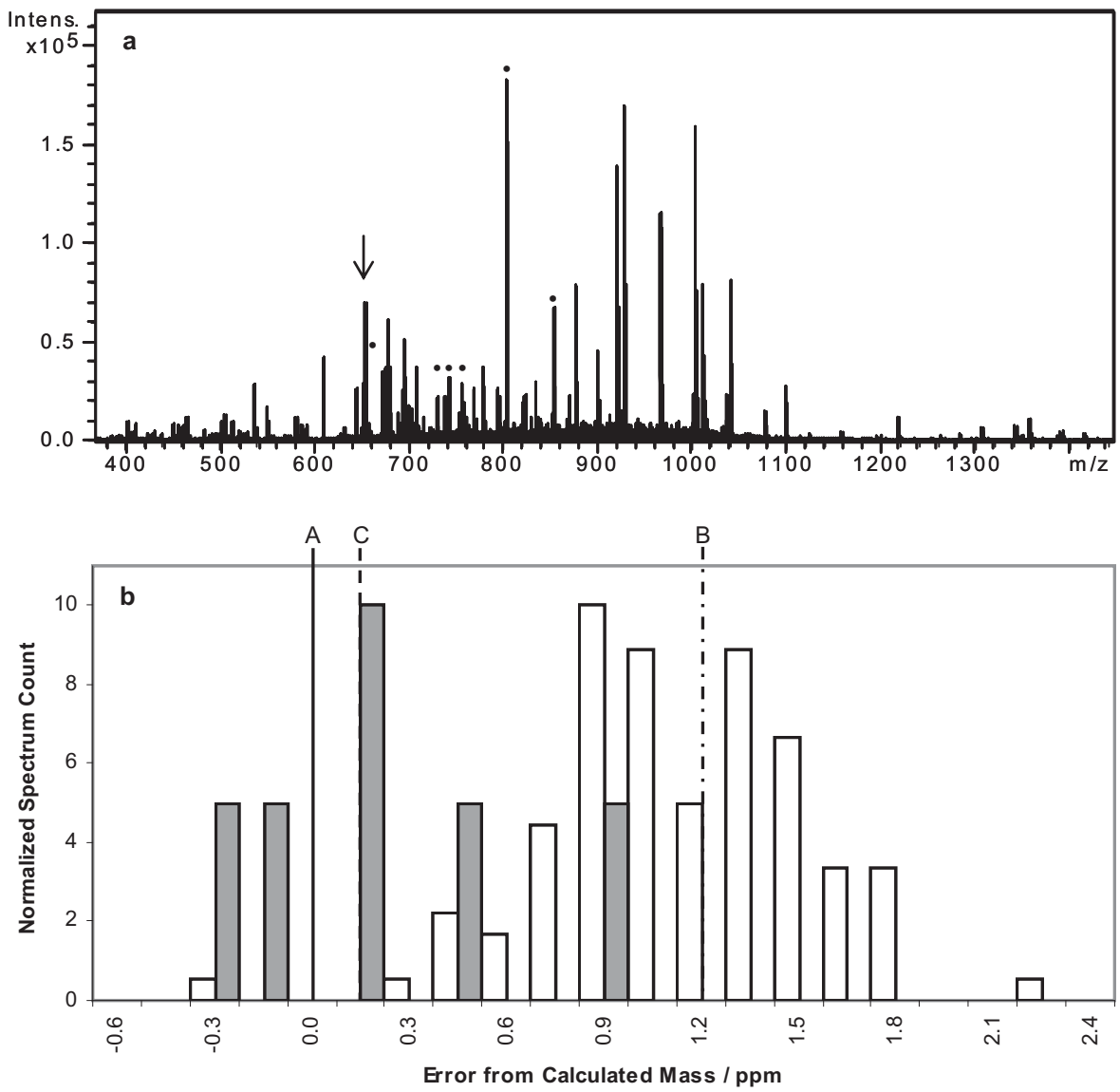

Figure 3. Recalibration performance analysis from protein mixture dataset no. 4. (a) Averaged mass spectrum (3 min observation time, 44 individual spectra) with calibrant ions identified from MS/MS analysis marked with $(\bullet)$ and a peak identified by the MMF search as the $2+$ ion of an 11 residue peptide from BSA marked with $(\downarrow)$. (b) Error analysis on the $2+$ ion. A perfect measurement of the mass would be at $0 \mathrm{ppm}$ (line marked " $\mathrm{A}$ "). Open bars are a histogram of the individual mass values obtained from all raw mass spectra where this isotopic distribution was detected. The line marked " $\mathrm{B}$ " is the average of these values. Shaded bars are a histogram of the mass values obtained from the averaged and recalibrated mass spectra produced from the DASER analysis. The line marked " $\mathrm{C}$ " is the average of these values.

of peptide hits and sequence coverage for each protein in the mixture (Table 3) were similar to those found for dataset no. 4. The expectation values show that the top three hits (BSA, transferrin, and alcohol dehydrogenase) are well above the stochastic distribution boundary. The next two hits (lysozyme and $\beta$-galactosidase) are right on this boundary despite their scores falling above the $0.3 \%$ false positive rate cutoff. The next highest hit is cytochrome $c$ although with this sample's lower loading, both its score and expectation value indicate that this small protein is not confidently identified. The large gap in expectation value between cytochrome $c$ and lysozyme and $\beta$-galactosidase is a further indication that this is where the significance cutoff should be placed.

\section{Complex Human Blood Serum Sample}

Blood serum from a CF patient that was isotopically labeled for relative quantitation was used to assess the performance of the DASER algorithm when applied to a complex mixture. The same CF sample was analyzed via 2D-LC-MS/MS five consecutive times over the course of approximately $5 \mathrm{wk}$. The analyses were interspersed with other experiments on this instrument and therefore represent the common instrumental variations that are expected to be observed in any laboratory setting. The MS/MS results from each chromatogram in the set making up an individual 2D-LC dataset were exported in MGF format and combined into a single file. The combined MGF file was submitted to Mascot for an MS/MS ion search (NCBI non-redundant database, Homo sapiens species filter, trypsin cleavage rules, two missed cleavages, methylated cysteine (C) fixed modification, light and heavy acetyl (K- and N-terminal) variable modifications, $10 \mathrm{ppm}$ mass accuracy).

As would be expected, each subsequent experiment identified some of the same proteins from previous experiments as well as some new proteins $[2,3]$. Overall, only 77 proteins were identified when combining all 
Table 3. Mascot and MMF search results from protein mixture dataset no. 2, $50 \mathrm{fmol}$ of each protein loaded on the column

\begin{tabular}{|c|c|c|c|c|c|c|c|c|c|c|c|c|}
\hline \multirow[b]{2}{*}{ Accession } & \multirow[b]{2}{*}{$\begin{array}{c}\text { Protein } \\
\text { description }\end{array}$} & \multicolumn{4}{|c|}{ Mascot } & \multicolumn{5}{|c|}{ MMF } & \multirow[b]{2}{*}{$\begin{array}{l}\text { Seq cov } \\
\text { increase }\end{array}$} & \multirow{2}{*}{$\begin{array}{l}\text { Seq cov } \\
\text { fold } \\
\text { increase }\end{array}$} \\
\hline & & Rank & Score $^{a}$ & $\begin{array}{l}\text { Total } \\
\text { hits }\end{array}$ & $\begin{array}{l}\text { Seq } \\
\text { cov }\end{array}$ & Rank & Score $^{b}$ & $\begin{array}{l}\text { Exp } \\
\text { value }\end{array}$ & $\begin{array}{l}\text { Total } \\
\text { hits }\end{array}$ & $\begin{array}{l}\text { Seq } \\
\text { cov }\end{array}$ & & \\
\hline AAN17824 & $\begin{array}{l}\text { Bos taurus } \\
\text { serum albumin }\end{array}$ & 2 & 45 & 2 & 4 & 1 & 13.5 & 4.2E-05 & 28 & 40 & 36 & 9.0 \\
\hline AAA96735 & $\begin{array}{l}\text { Bos taurus } \\
\text { transferrin }\end{array}$ & 1 & 45 & 2 & 3 & 2 & 12.6 & 0.0006 & 18 & 22 & 19 & 6.3 \\
\hline $\mathrm{LZCH}$ & $\begin{array}{l}\text { Lysozyme } c \\
\text { precursor }\end{array}$ & c & 35 & 2 & 19 & 4 & 10.1 & 1.1 & 4 & 33 & 14 & 0.7 \\
\hline GBEC & $\beta$-Galactosidase & - & 0 & 0 & 0 & 5 & 10.0 & 1.3 & 9 & 9 & 9 & $\operatorname{lnf}$ \\
\hline DEBYA & $\begin{array}{l}\text { Alcohol } \\
\text { dehydrogenase }\end{array}$ & - & 0 & 0 & 0 & 3 & 10.8 & 0.15 & 10 & 22 & 22 & $\operatorname{lnf}$ \\
\hline ССВО & $\begin{array}{l}\text { Cytochrome } c \\
\text { bovine }\end{array}$ & - & 0 & 0 & 0 & d & 9.5 & 6.2 & 3 & 23 & 23 & $\operatorname{lnf}$ \\
\hline \multicolumn{4}{|c|}{$\begin{array}{l}\text { Total hits/average seq cov (including } 0 \mathrm{~s} \text { ) } \\
\text { Fold increase }\end{array}$} & 6 & 4.3 & & & & $\begin{array}{l}72 \\
12.0\end{array}$ & $\begin{array}{l}25 \\
5.7\end{array}$ & & \\
\hline
\end{tabular}

a Significance cutoff for the Mascot search was a score of $40(P<0.05)$.

bignificance cutoff for the MMF search was a score of $10.0(0.3 \%$ false positive rate).

'Lysozyme was below the Mascot significance level even though two peptide hits were identified as being associated with this protein.

${ }^{d}$ Cytochrome $c$, while the next highest ranked hit after $\beta$-galactosidase, is not significant until the false positive rate is increased to $2 \%$.

e Total hits and average sequence coverage calculations include hits from all proteins even if the actual protein score was below the confidence level

for a given search.

of the results. The low number of identifications is not an uncommon observation with LC-MS/MS experiments performed on FTMS instruments and is likely due to the relatively low MS/MS acquisition rate compared with quadrupole or linear ion trap instruments. The Mascot MS/MS ion search results were then used to recalibrate the data using the DASER algorithm. A summary of the recalibration results for each of the five datasets is shown in Table 4. A much greater proportion of data is recalibrated in the serum samples (average of $53 \%$ ) than in the simple protein mixture samples (average of $21 \%$, Table 1 ). The reason for this is believed to be the additional peptides available within the analysis to collect MS/MS data on. This yields additional MS/MS spectra submitted to Mascot even when accounting for the additional run time of these 2D versus $1 \mathrm{D}$ experiments. Several individual chromatograms (from the more populated salt fractions) gave greater than $80 \%$ recalibration coverage.

Despite having many more spectra included in the recalibrated set, the serum samples demonstrated recalibration performances similar to the protein mixtures. The original average RMS error was over $4 \mathrm{ppm}$ and the standard deviation was almost 1 ppm. After recalibration, the average RMS and standard deviations of the errors are both below $0.5 \mathrm{ppm}$, indicating that the mass list produced from the recalibrated spectra is accurate to $\sim 1$ ppm. The large initial mass errors for the serum datasets is likely representative of the larger range in space charge conditions exhibited in the ICR cell during the 2D-LC elution of these complex samples. The effect of space charge on the mass accuracy is demonstrated in Figure 4. The total ion current (TIC) along with the original and recalibrated mass errors for each averaged spectrum in serum dataset no. 4 is shown. The correlation between the original mass errors and the TIC is quite striking. Although the recalibrated error trace shows some of the TIC trace features, indicating that the effects of space charge have not been completely eliminated, the absolute mass error as well as its variability has been greatly reduced. Table 1 also shows that while the initial RMS error for dataset no. 1 is greater than 7 ppm, the recalibration is still able to function properly and reduce the error down to $0.33 \mathrm{ppm}$. Despite the initial quality of the instrument or external calibration, the DASER method is able to give consistently high

Table 4. Recalibration results from human serum datasets (each representing 11 salt fractions or LC-MS/MS datasets)

\begin{tabular}{|c|c|c|c|c|c|c|c|c|c|c|}
\hline \multirow[b]{2}{*}{ Dataset } & \multirow[b]{2}{*}{$\begin{array}{c}\text { No. MS/MS } \\
\text { spectra }\end{array}$} & \multirow[b]{2}{*}{$\begin{array}{c}\% \mathrm{MS} / \mathrm{MS} \\
\text { spectra IDed }\end{array}$} & \multirow[b]{2}{*}{$\begin{array}{c}\% \text { Data } \\
\text { recalibrated }\end{array}$} & \multicolumn{6}{|c|}{ Errors } & \multirow[b]{2}{*}{$\begin{array}{l}\text { Unique } \\
\text { masses }\end{array}$} \\
\hline & & & & Orig RMS & $\begin{array}{l}\text { Recal } \\
\text { RMS }\end{array}$ & Orig mean & $\begin{array}{l}\text { Recal } \\
\text { mean }\end{array}$ & Orig STD & $\begin{array}{l}\text { Recal } \\
\text { STD }\end{array}$ & \\
\hline 1 & 1192 & $23 \%$ & $55 \%$ & 7.20 & 0.33 & 7.05 & 0.00 & 1.08 & 0.38 & 22406 \\
\hline 2 & 936 & $38 \%$ & $74 \%$ & 1.28 & 0.47 & 0.59 & 0.00 & 1.00 & 0.52 & 20751 \\
\hline 3 & 722 & $36 \%$ & $45 \%$ & 2.58 & 0.28 & 2.45 & 0.00 & 0.76 & 0.32 & 13564 \\
\hline 4 & 969 & $23 \%$ & $44 \%$ & 5.21 & 0.42 & 5.10 & 0.00 & 0.93 & 0.47 & 18255 \\
\hline 5 & 1003 & $27 \%$ & $48 \%$ & 4.51 & 0.49 & 4.38 & 0.00 & 1.07 & 0.56 & 21439 \\
\hline Averages & & $29 \%$ & $53 \%$ & 4.15 & 0.40 & 3.91 & 0.00 & 0.97 & 0.45 & \\
\hline
\end{tabular}



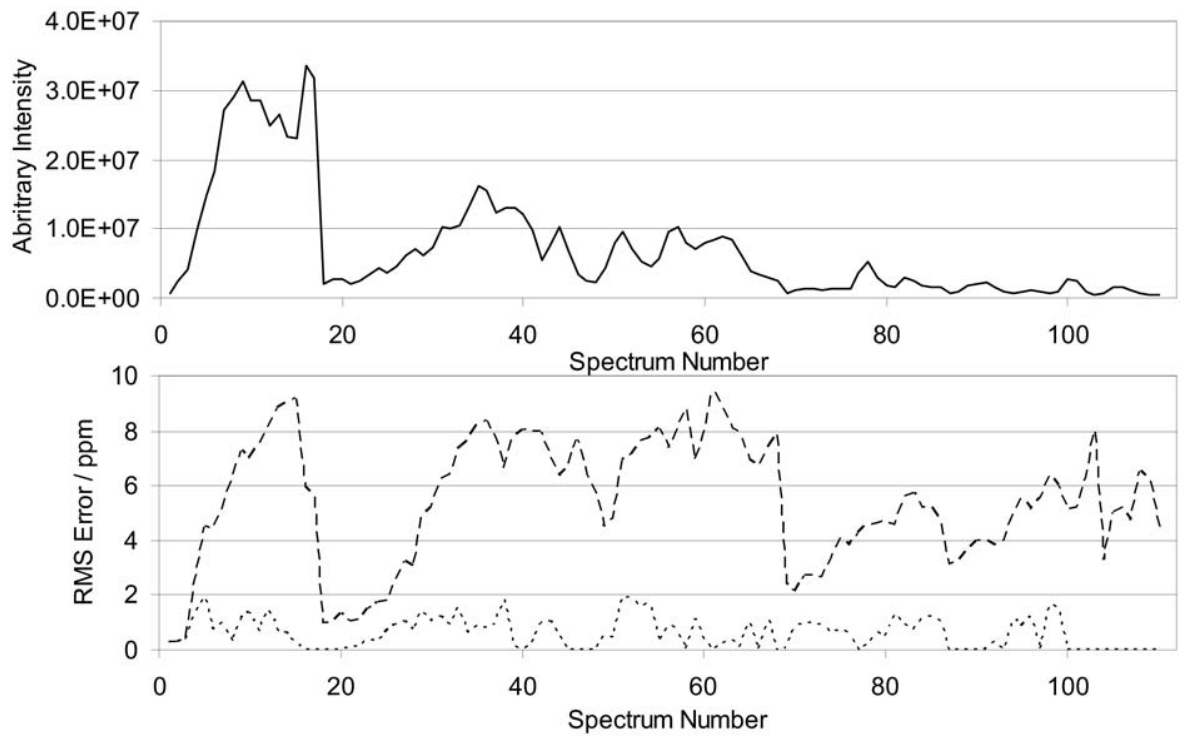

Figure 4. Total ion current (TIC) (solid), original (dashed), and recalibrated (dotted) mass error traces for human serum dataset no. 4 .

performance, approaching the best reported values for mass measurement accuracy [14, 18, 32].

One of the major motivations driving development of the DASER-MMF technique was to provide a tool for enhancing identification of low abundance proteins from highly complex mixtures of clinical or biological importance, such as the serum samples used in this work. While the DASER performance on the serum datasets is quite impressive, the MMF search was only able to positively identify one protein (HSA) in these very complex mixtures. In contrast, Mascot was able to identify over 20 proteins from a single experiment. The reason for the poor MMF search performance is believed to be due to the large number of peaks identified in the 2D datasets (last column in Table 4) as well as the number of variable modifications that had to be considered due to the isotopic labeling. These two factors lead to additional random matches to the decoy database search, which increases the protein score cutoff value. While accurate mass was enough to identify proteins in a simple mixture, it is not adequate to handle very complex mixtures. Additional discriminating factors are needed to further enhance the identification abilities of the MMF Search.

As the peptides within this serum sample were isotopically labeled, every peptide shows up as a doublet of a "light" and "heavy" peak. An additional processing step was developed to create a reduced list of masses with just the light label present, and to associate with these masses a count of the number of lysines present in the peptide. The acetyl label will attach to lysine residues as well as the N-terminus of the peptide (of which there is always only one). The number of lysine residues present in the peptide was then used as an additional discriminating factor within the MMF search. The additional processing reduced the peak list sizes by $10-20$ times and enabled identification of 11 proteins from a single experiment at a false positive rate of $0.5 \%$ (six proteins at $0.3 \%$ ). Nine of the proteins were also identified by Mascot (six of the top seven Mascot hits were mutually identified). While the MMF search still does not show significant improvements over the performance of the Mascot MS/MS based search, clearly adding additional discriminating factors to the search is a beneficial direction to pursue.

\section{Future Developments}

As the lysine count was able to increase the identification power from 1 to 11 proteins, current work is underway to add additional discriminating factors to the MMF search algorithm. Specifically, peptide retention time is being considered as a second discriminating factor for the MMF search. There are several published methods for prediction of retention time based on peptide sequence [33-36], and adding this factor has proven useful in other accurate mass based search schemes $[37,38]$. As the observed mass is already a very accurate factor within the MMF search, extreme accuracy in the retention time is likely not necessary, and the published methods should provide sufficient performance (the order of minutes should be sufficient). Preliminary results indicate that addition of crude retention time prediction to the MMF search allows three times as many proteins to be identified within a given search.

As the MMF search deals with peptide masses, it has unique applicability to peptide and spectral count based relative quantitation techniques [39-41]. The MMF search has the ability to identify 10 times as many peptides from a given protein versus similar MS/MS based search schemes, allowing many more peptides to 
be counted, and potentially making this measure more accurate and useful. This could allow a single dataset to be used to identify and quantitate proteins present in complex mixtures.

\section{Conclusions}

Although the DASER-MMF technique has been shown here applied to FTMS based datasets, it is not specific to a particular type of mass analyzer. This method can be easily implemented on data from any instrument capable of higher mass accuracy, such as Orbitrap (Thermo Electron Corp., Bremen, Germany) and TOF based mass spectrometers. Additionally, the MS/MS data does not have to be high-resolution; it only has to be correlated to a parent ion in the high-resolution MS spectra. The DASER method relies on information already contained in the LC-MS/MS dataset that was not efficiently used by other processing techniques. Implementation is accomplished through post-processing the dataset and therefore does not require altering the instrument hardware or software control. As such, the dynamic range and overall sampling rate of the instrument is unchanged, in contrast to other methods for improving mass accuracy, all of which compromise these performance metrics. The fact that the instrument dynamic range is not compromised is illustrated by the fact that several of the complex serum datasets presented had RMS mass errors of over $4 \mathrm{ppm}$. This is high for a 12 tesla FTMS instrument and is reflective of the varying ICR cell loadings that occurred as no effort was made to limit the number of ions accumulated in a given acquisition sequence. Despite the large original mass errors and the overall complexity of the datasets, RMS mass errors and standard deviations of less than $0.5 \mathrm{ppm}$ were routinely achieved, with some errors in the $0.25 \mathrm{ppm}$ range.

The high mass accuracy afforded by the DASER method is uniquely applicable to the MMF search scheme. The MMF search has been shown to identify proteins (often small or of low intensity) missed by standard MS/MS based search methods. The MMF search is exceptional for its ability to identify proteins present in a mixture using only peptide mass values. Searches of large protein databases (over two million sequences) were able to produce IDs of all the proteins within the mixture at very low false positive rates (less than $0.5 \%$ ). While other accurate mass-only search methods have been able to identify multiple proteins within a mixture, all of the previous reports have used much smaller databases (100s or 1000 s or proteins) $[4,5$, $25,37]$. The low false positive rates producing complete mixture IDs while searching a database of this size are very unique.

The software programs (Microsoft Windows executables) used to implement the DASER and MMF techniques are available for download at www. proteincentre.com.

\section{Acknowledgments}

The authors acknowledge funding of this work by a gift from an anonymous donor to support research in proteomics at UNC, NIH grants P30 CA0-16,086, 1S10RR16776, caBIG-ICR-07-20-01, and P30 Es010126, Cystic Fibrosis Foundation grant CFFTI BORCHE05U0, startup funds from the University of Victoria, funding from Genome Canada and Genome British Columbia, and collaboration with Bruker Daltonics. The authors also acknowledge the use of the 12-tesla FTICR mass spectrometer at the UNC-Duke Michael Hooker Proteomics Center, NC (NIH 1-S10RR019889-01 and NCBC 2005-IDG-1015).

\section{References}

1. Washburn, M. P.; Wolters, D.; Yates, J. R. III. Large-Scale Analysis of the Yeast Proteome by Multidimensional Protein Identification Technology. Nat. Biotechnol. 2001, 19, 242-247.

2. Kislinger, T.; Gramolini, A. O.; MacLennan, D. H.; Emili, A. Multidimensional Protein Identification Technology (MudPIT): Technical Overview of a Profiling Method Optimized for the Comprehensive Proteomic Investigation of Normal and Diseased Heart Tissue. J. Am. Soc. Mass Spectrom. 2005, 16, 1207-1220.

3. Liu, H.; Sadygov, R. G.; Yates, J. R. III. A Model for Random Sampling and Estimation of Relative Protein Abundance in Shotgun Proteomics. Anal. Chem. 2004, 76, 4193-4201.

4. Shen, Y.; Tolic, N.; Zhao, R.; Pasa-Tolic, L.; Li, L.; Berger, S. J.; Harkewicz, R.; Anderson, G. A.; Belov, M. E.; Smith, R. D. HighThroughput Proteomics Using High-Efficiency Multiple-Capillary Liquid Chromatography with On-Line High-Performance ESI FTICR Mass Spectrometry. Anal. Chem. 2001, 73, 3011-3021.

5. Smith, R. D.; Anderson, G. A.; Lipton, M. S.; Pasa-Tolic, L.; Shen, Y.; Conrads, T. P.; Veenstra, T. D.; Udseth, H. R. An Accurate Mass Tag Strategy for Quantitative and High-Throughput Proteome Measurements. Proteomics 2002, 2, 513-523.

6. Pasa-Tolic, L.; Masselon, C.; Barry, R. C.; Shen, Y.; Smith, R. D. Proteomic Analyses Using an Accurate Mass and Time Tag Strategy. Biotechniques 2004, 37, 621-624, 626-633, 636 passim.

7. Goodlett, D. R.; Bruce, J. E.; Anderson, G. A.; Rist, B.; Pasa-Tolic, L.; Fiehn, O.; Smith, R. D.; Aebersold, R. Protein Identification with a Single Accurate Mass of a Cysteine-Containing Peptide and Constrained Database Searching. Anal. Chem. 2000, 72, 1112-1118.

8. Marshall, A. G.; Hendrickson, C. L.; Jackson, G. S. Fourier Transform Ion Cyclotron Resonance Mass Spectrometry: A Primer. Mass Spectrom. Rev. 1998, 17, 1-35.

9. Ledford, E. B.; Jr.; Rempel, D. L.; Gross, M. L. Space Charge Effects in Fourier Transform Mass Spectrometry. Mass Calibration. Anal. Chem. 1984, 56, 2744-2748.

10. Easterling, M. L.; Mize, T. H.; Amster, I. J. Routine Part-per-Million Mass Accuracy for High-Mass Ions: Space-Charge Effects in MALDI FT-ICR. Anal. Chem. 1999, 71, 624-632.

11. Belov, M. E.; Zhang, R.; Strittmatter, E. F.; Prior, D. C.; Tang, K.; Smith, R. D. Automated Gain Control and Internal Calibration with External Ion Accumulation Capillary Liquid Chromatography-Electrospray Ionization Fourier Transform Ion Cyclotron Resonance. Anal. Chem. 2003, $75,4195-4205$.

12. Belov, M. E.; Rakov, V. S.; Nikolaev, E. N.; Goshe, M. B.; Anderson, G. A.; Smith, R. D. Initial Implementation of External Accumulation Liquid Chromatography/Electrospray Ionization Fourier Transform Ion Cyclotron Resonance with Automated Gain Control. Rapid Commun. Mass Spectrom. 2003, 17, 627-636.

13. Syka, J. E.; Marto, J. A.; Bai, D. L.; Horning, S.; Senko, M. W.; Schwartz, J. C.; Ueberheide, B.; Garcia, B.; Busby, S.; Muratore, T.; Shabanowitz, J.; Hunt, D. F. Novel Linear Quadrupole Ion Trap/FT Mass Spectrometer Performance Characterization and Use in the Comparative Analysis of Histone H3 Post-Translational Modifications. J. Proteome Res. 2004, 3 , 621-626.

14. Burton, R. D.; Matuszak, K. P.; Watson, C. H.; Eyler, J. R. Exact Mass Measurements Using a 7 Tesla Fourier Transform Ion Cyclotron Resonance Mass Spectrometer in a Good Laboratory Practices-Regulated Environment. J. Am. Soc. Mass Spectrom. 1999, 10, 1291-1297.

15. O'Connor, P. B.; Costello, C. E. Internal Calibration on Adjacent Samples (InCAS) With Fourier Transform Mass Spectrometry. Anal. Chem. 2000, 72, 5881-5885.

16. Hannis, J. C.; Muddiman, D. C. A Dual Electrospray Ionization Source Combined with Hexapole Accumulation to Achieve High Mass Accuracy of Biopolymers in Fourier Transform Ion Cyclotron Resonance Mass Spectrometry. J. Am. Soc. Mass Spectrom. 2000, 11, 876-883.

17. Lee, S. W.; Berger, S. J.; Martinovic, S.; Pasa-Tolic, L.; Anderson, G. A. Shen, Y.; Zhao, R.; Smith, R. D. Direct Mass Spectrometric Analysis of Intact Proteins of the Yeast Large Ribosomal Subunit Using Capillary LC/FTICR. Proc. Natl. Acad. Sci. U.S.A. 2002, 99, 5942-5947.

18. Nepomuceno, A. I.; Muddiman, D. C.; H. Robert Bergen, I.; Craighead J. R.; Burke, M. J.; Caskey, P. E.; Allan, J. A. Dual Electrospray Ionization Source for Confident Generation of Accurate Mass Tags Using Liquid 
Chromatography Fourier Transform Ion Cyclotron Resonance Mass Spectrometry. Anal. Chem. 2003, 75, 3411-3418.

19. Bruce, J. E.; Anderson, G. A.; Brands, M. D.; Pasa-Tolic, L.; Smith, R. D. Obtaining More Accurate Fourier Transform Ion Cyclotron Resonance Mass Measurements Without Internal Standards Using Multiply Charged Ions. J. Am. Soc. Mass Spectrom. 2000, 11, 416-421.

20. Kaiser, N. K.; Anderson, G. A.; Bruce, J. E. Improved Mass Accuracy for Tandem Mass Spectrometry. J. Am. Soc. Mass Spectrom. 2005, 16, 463-470.

21. Wu, S.; Kaiser, N. K.; Meng, D.; Anderson, G. A.; Zhang, K.; Bruce, J. E. Increased Protein Identification Capabilities through Novel Tandem MS Calibration Strategies. J. Proteome Res. 2005, 4, 1434-1441.

22. Wong, R. L.; Amster, I. J. Sub Part-Per-Million Mass Accuracy by Using Stepwise-External Calibration in Fourier Transform Ion Cyclotron Resonance Mass Spectrometry. J. Am. Soc. Mass Spectrom. 2006, 17, 16811691.

23. Tolmachev, A. V * Monroe, M. E.; Jaitly, N.; Petyuk, V. A.; Adkins, J. N.; Smith, R. D. Mass Measurement Accuracy in Analyses of Highly Complex Mixtures Based Upon Multidimensional Recalibration. Anal. Chem. 2006, 78, 8374-8385.

24. Palmblad, M.; Bindschedler, L. V.; Gibson, T. M.; Cramer, R. Automatic Internal Calibration in Liquid Chromatography/Fourier Transform Ion Cyclotron Resonance Mass Spectrometry of Protein Digests. Rapid Commun. Mass Spectrom. 2006, 20, 3076-3080.

25. Ramström, M.; Palmblad, M.; Markides, K. E.; Håkansson, P.; Bergquist, J. Protein Identification in Cerebrospinal Fluid Using Packed Capillary Liquid Chromatography Fourier Transform Ion Cyclotron Resonance Mass Spectrometry. Proteomics 2003, 3, 184-190.

26. Francl, T. J.; Sherman, M. G.; Hunter, R. L.; Locke, M. J.; Bowers, W. D.; McIver, R. T. Experimental Determination of the Effects of Space Charge on Ion Cyclotron Resonance Frequencies. Int. J. Mass Spectrom. Ion Processes 1983, 54, 189-199.

27. Taylor, P. K.; Amster, I. J. Space Charge Effects on Mass Accuracy for Multiply Charged Ions in ESI-FTICR. Int. J. Mass Spectrom. 2003, 222, 351-361.

28. Henzel, W. J.; Watanabe, C.; Stults, J. T. Protein Identification: The Origins of Peptide Mass Fingerprinting. J. Am. Soc. Mass Spectrom. 2003, 14, 931-942.

29. Pappin, D. J. C.; Hojrup, P.; Bleasby, A. J. Rapid Identification of Proteins by Peptide-Mass Fingerprinting. Curr. Biol. 1993, 3, 327-332.

30. Fenyo, D.; Beavis, R. C. A Method for Assessing the Statistical Significance of Mass Spectrometry-Based Protein Identifications Using General Scoring Schemes. Anal. Chem. 2003, 75, 768-774.
31. Keller, A.; Nesvizhskii, A. I.; Kolker, E.; Aebersold, R. Empirical Statistical Model to Estimate the Accuracy of Peptide Identifications Made by MS/MS and Database Search. Anal. Chem. 2002, 74, 5383-5392.

32. Rodgers, R. P.; White, F. M.; Hendrickson, C. L.; Marshall, A. G.; Andersen, K. V. Resolution, Elemental Composition, and Simultaneous Monitoring by Fourier Transform Ion Cyclotron Resonance Mass Spectrometry of Organosulfur Species Before and After Diesel Fuel Processing. Anal. Chem. 1998, 70, 4743-4750.

33. Casal, V.; Martin-Alvarez, P. J.; Herraiz, T. Comparative Prediction of the Retention Behavior of Small Peptides in Several Reversed-Phase High-Performance Liquid Chromatography Columns by Using Partial Least Squares and Multiple Linear Regression. Anal. Chim. Acta 1996 326, 77-84.

34. Palmblad, M.; Ramstrom, M.; Markides, K. E.; Hakansson, P.; Bergquist, J. Prediction of Chromatographic Retention and Protein Identification in Liquid Chromatography/Mass Spectrometry. Anal. Chem. 2002, 74 5826-5830.

35. Petritis, K.; Kangas, L. J.; Ferguson, P. L.; Anderson, G. A.; Pasa-Tolic, L. Lipton, M. S.; Auberry, K. J.; Strittmatter, E. F.; Shen, Y.; Zhao, R.; Smith, R. D. Use of Artificial Neural Networks for the Accurate Prediction of Peptide Liquid Chromatography Elution Times in Proteome Analyses. Anal. Chem. 2003, 75, 1039-1048.

36. Krokhin, O. V.; Craig, R.; Spicer, V.; Ens, W.; Standing, K. G.; Beavis, R. C.; Wilkins, J. A. An Improved Model for Prediction of Retention Times of Tryptic Peptides in Ion Pair Reversed-Phase HPLC: Its Application to Protein Peptide Mapping by Off-Line HPLC-MALDI MS Mol. Cell. Proteom. 2004, 3, 908-919.

37. Nilsson, S.; Ramstrom, M.; Palmblad, M.; Axelsson, O.; Bergquist, J. Explorative Study of the Protein Composition of Amniotic Fluid by Liquid Chromatography Electrospray Ionization Fourier Transform Ion Cyclotron Resonance Mass Spectrometry. J. Proteome Res. 2004, 3, $884-889$.

38. Norbeck, A. D.; Monroe, M. E.; Adkins, J. N.; Anderson, K. K.; Daly, D. S.; Smith, R. D. The Utility of Accurate Mass and LC Elution Time Information in the Analysis of Complex Proteomes. J. Am. Soc. Mass Spectrom. 2005, 16, 1239-1249.

39. Pang, J. X.; Ginanni, N.; Dongre, A. R.; Hefta, S. A.; Opiteck, G. J. Biomarker Discovery in Urine by Proteomics. J. Proteome Res. 2002, 1, $161-169$.

40. Gao, J.; Opiteck, G. J.; Friedrichs, M. S.; Dongre, A. R.; Hefta, S. A. Changes in the Protein Expression of Yeast as a Function of Carbon Source. J. Proteome Res. 2003, 2, 643-649.

41. Liu, H.; Sadygov, R. G.; Yates, J. R. A Model for Random Sampling and Estimation of Relative Protein Abundance in Shotgun Proteomics. Anal. Chem. 2004, 76, 4193-4201. 